\title{
Ne craignez pas d'ouvrir un cabinet médical!
}

Jürgen Bachmann

Correspondance:

Jürgen Bachmann

Bachmann Treuhand AG

FMH Treuhand Services

Am Schanzengraben 25

CH-8002 Zurich
«Mon cabinet a-t-il des chances de survivre? Estce que je ne présume pas trop de mes forces? Puis-je financer tout cela? Ces investissements me font peur!» - Nous entendons ces réflexions et bien d'autres encore pratiquement lors de chaque ouverture ou reprise de cabinet planifiée ou en cours d'évaluation. Ce pas vers l'indépendance est en partie aussi un pas vers l'inconnu. Or, dans la plupart des cas, il s'avère positif. Dans les lignes qui suivent, nous aimerions vous aider à surmonter vos craintes éventuelles et vous montrer comment réduire les risques afin que votre démarche soit une réussite!

\section{Premières réflexions}

Au début, il y a l'idée. Mais, souvent, bien des années s'écoulent entre l'idée qui germe et sa réalisation. Si vous répondez aux critères suivants, il vaut la peine d'examiner plus en détail l'idée d'ouvrir ou de reprendre un cabinet médical. Vous disposez:

- du savoir-faire nécessaire et d'une compétence élevée dans votre discipline médicale.

Vous avez:

- le courage de remettre vos idées en question;

- le courage de les réaliser;

- une bonne dose d'énergie, l'envie de créer;

- du talent pour planifier et organiser;

- une saine portion d'optimisme;

- la volonté de tenir bon.

Vous savez:

- aborder les gens de manière ouverte;

- négocier et convaincre.

Vous êtes prêt/e:

- à vous engager et

- à tirer les leçons de vos erreurs.

\section{Ai-je le profil d'un entrepreneur?}

Le passage à l'indépendance professionnelle doit être préparé le plus sérieusement possible. En répondant honnêtement aux questions suivantes, vous disposerez d'une base saine pour prendre votre décision initiale:

- Pourquoi est-ce que je désire être indépendant? Quels sont mes objectifs personnels et matériels?

- Quelles capacités particulières me donnentelles l'assurance que je peux créer ou reprendre un cabinet médical de mes propres forces et le gérer avec succès?
- En quoi mon réseau de relations me sera-t-il utile?

- Ai-je la personnalité d'un gagnant, d'un entrepreneur? Les gens me sont-ils sympathiques, ai-je de bons contacts avec eux? Suis-je en mesure de gérer mes ressources de manière judicieuse? Est-ce que je garde la vue d'ensemble dans les situations perturbées? Suis-je en mesure de fixer des priorités? Suis-je capable de me motiver et de supporter sereinement les revers?

- Quelles sont mes capacités financières? Ai-je suffisamment de réserves? Que peut-il nous arriver, à moi et ma famille, si je gagne moins que maintenant pendant un certain temps, ou que je dois puiser dans mes fonds propres?

- Que pense mon entourage (partenaire, famille, amis) de mes plans? Est-il prêt à me soutenir si, un jour, tout ne tourne pas parfaitement rond?

- Ai-je un plan d'affaires qui tient la route, aussi du point de vue des experts (banques, conseiller fiscal, etc.)?

- Suis-je physiquement et moralement en bonne forme? Ai-je l'énergie et la motivation de m'engager dans une nouvelle aventure?

- Quelles sont les alternatives à ma disposition si mes plans échouent? Suis-je assez souple mentalement pour supporter un échec éventuel?

\section{Mon analyse est-elle correcte?}

Trois choses sont essentielles pour ouvrir ou reprendre un cabinet médical avec succès:

- une auto-évaluation réaliste;

- une évaluation réaliste du contexte général et de ses aspects économiques;

- la disponibilité de travailler beaucoup pendant quelques années et, suivant les circonstances, de gagner moins durant un certain temps.

Si cet «auto-examen» vous permet de conclure que vous serez à même de gérer un cabinet en propre, il vous faut maintenant répondre aux questions suivantes:

\section{Reprise d'un cabinet ou nouvelle installation?}

L'expérience et la logique montrent qu'il est plus simple de reprendre un cabinet que d'en créer un, et plus facile d'évaluer les risques d'une reprise que d'une nouvelle installation. En revanche, l'ouverture d'un nouveau cabinet laisse une plus grande marge de manœuvre. Mais souvent, la situation ne vous laisse pas le choix. 


\section{Admission à pratiquer et emplacement du cabinet}

Ces deux points sont en lien direct tant avec la reprise que l'ouverture d'un cabinet; certaines contraintes sont incontournables selon la situation. Quoi qu'il en soit, la question de l'emplacement doit être clarifiée suffisamment tôt. Examinez vos intentions avec la société cantonale de médecine et la direction de la santé concernée. Vous trouverez les adresses et personnes de contact utiles sous www.fmh.ch.

\section{Cabinet au domicile ou à l'extérieur?}

Un cabinet au lieu de domicile présente l'avantage du bref déplacement. L'inconvénient réside dans la difficulté à séparer activité professionnelle et vie privée. A cet égard, vos préférences personnelles et celles de votre conjoint ou partenaire jouent un rôle décisif.

\section{Location ou propriété privée?}

Lors de la reprise d'un cabinet, cette question est définie par l'offre disponible. La propriété privée lie beaucoup plus que la location et influe grandement sur le montant des fonds initiaux nécessaires; elle présente généralement un plus grand risque que la location.

\section{Préparation personnelle}

En étant libre praticien, vous dépendez uniquement de vous-même. C'est une expérience fascinante et après l'avoir vécue, vous ne voudrez plus y renoncer. Néanmoins, vous ne disposerez plus d'un service opérationnel complet en matière de

Figure 1

Phases du projet «Mon propre cabinet».

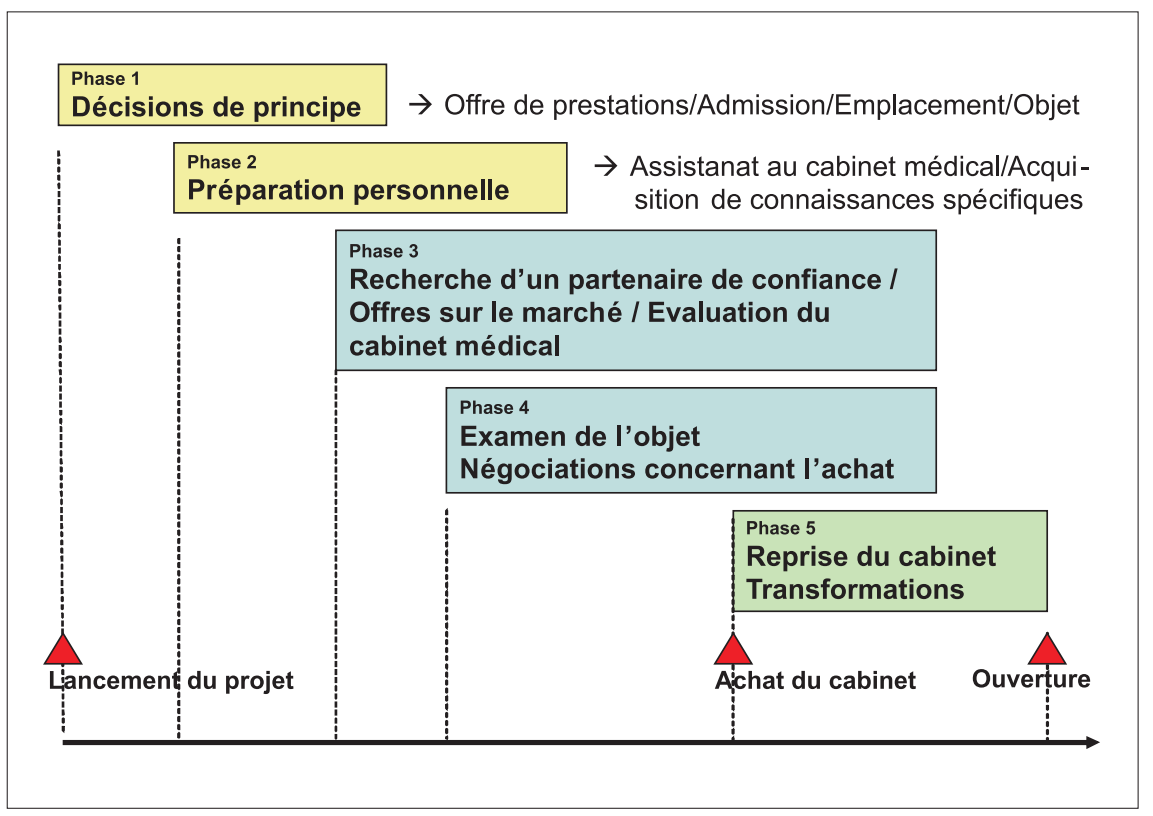

logistique, matériel, personnel et finances; vous devrez dorénavant vous soucier vous-même de tous ces aspects. Avant de vous mettre à votre compte, nous vous conseillons vivement d'effectuer un assistanat de quelques mois au cabinet médical envisagé, après avoir quitté votre statut d'employé. Vous pourrez ainsi, sous supervision, faire des expériences dans votre futur domaine d'activité et renforcer votre décision quant à une future activité indépendante.

La durée totale entre le lancement du projet et l'ouverture du cabinet est généralement de 12 à 15 mois (fig. 1). Elle dépend dans une grande mesure de vos connaissances professionnelles et de votre expérience, de la situation du marché et des attentes de celle ou celui qui remet son cabinet.

\section{Evaluation du cabinet médical}

Ensuite ou parallèlement à ces préparatifs, il vous faut chercher un cabinet approprié, l'examiner, l'évaluer et procéder au choix, faire expertiser le bien immobilier et négocier l'achat. Ces démarches complexes ne font pas l'objet du présent article, mais doivent être discutées séparément de manière approfondie. Pour ce faire, vous avez notamment besoin de partenaires de confiance compétents. FMH Services, l'organisation de services du corps médical, vous offre son aide dans plusieurs domaines tels que la recherche d'un cabinet et son évaluation, l'étude et l'établissement d'un contrat, ainsi que des séminaires sur l'ouverture d'un cabinet ou une brochure spécialisée sur la reprise de cabinet.

\section{Viabilité économique et financement}

Cette question, d'importance existentielle, provoque souvent des craintes infondées, qui se dissipent lorsque toute la clarté est faite sur les implications financières de la situation envisagée. Pour cela, vous avez besoin d'un plan financier sérieux.

Ce plan aura pour point de départ le prix d'achat du cabinet à reprendre ou les investissements à réaliser pour l'ouverture d'un nouveau cabinet. Fort de ces indications ainsi que des données disponibles quant au passé du cabinet et aux possibilités de financement par des capitaux propres et étrangers, l'expert-comptable spécialisé dans la gestion de cabinets médicaux (p. ex. FMH Fiduciaire Services) à qui vous ferez appel établira une prévision modèle pour les deux à quatre prochaines années. Ce document contiendra vos budgets annuels ainsi que des plans de financement; il fera partie de votre plan d'affaires.

Vous définirez avec votre expert-comptable les conditions générales telles que le nombre de patients, le taux de travail, le chiffre d'affaires 
moyen, etc. Le calcul de variantes moins avantageuses ou plus favorables est possible et utile. Le plan d'affaires ainsi établi répondra à la question de la viabilité économique votre entreprise.

\section{Financement}

Vos réserves financières devraient pouvoir résister à une évolution éventuellement défavorable au cours des deux à trois premières années. Le besoin de financement, à définir sur la base du plan d'affaires, devrait comporter, en fonction du capital propre à disposition, une réserve appropriée incluse dans le calcul du financement étranger nécessaire. Il est impératif de régler la question du financement avant de procéder à l'achat ou de s'engager dans des investissements. Relevons que des moyens propres seront nécessaires même lors d'une évaluation positive par des tiers. Les instruments de financement suivants sont à disposition:

- capital propre: économies, héritage anticipé, retrait anticipé de capital du fonds de prévoyance professionnelle;

- diverses possibilités financières pour consolider la base de capital propre: augmentation de l'hypothèque sur l'immeuble que vous possédez éventuellement, avance sur titres et/ou polices d'assurance, prêts familiaux. Ces financements sont souvent plus avantageux que le recours à un capital étranger;

- crédit d'exploitation: il sert à financer le capital de roulement (matériel, médicaments, créances de patients), généralement sous forme de comptes courants. Il est souvent accordé sans garantie jusqu'à une certaine limite. Les intérêts sont plus élevés que ceux d'autres crédits mais ils peuvent toutefois être réduits selon les garanties fournies. Avantage essentiel: des intérêts ne sont exigibles qu'en cas d'utilisation du crédit;

- avance à terme fixe: elle est souvent accordée en combinaison avec un crédit d'exploitation. Avantage: intérêt réduit pour la part fixe d'un crédit d'exploitation dont on est sûr de faire usage;

- crédit d'investissement: sert à financer le capital immobilisé (installations, appareils). Souvent avec des quotes-parts d'amortissement à long terme;

- crédit-bail (leasing): comme alternative au crédit d'investissement. Le règlement des mensualités de crédit-bail génère des coûts. Avantage: moins de crédits nécessaires, les liquidités sont préservées, financement éventuellement plus avantageux que les crédits. Désavantage: la propriété des biens reste chez le bailleur de fonds, coûts éventuellement plus élevés que ceux des crédits;
- affacturage: avances sur paiements des patients par des services de recouvrement externes.

Ce sont là les possibilités de financement les plus importantes. D'autres possibilités plus affinées pourront apparaître dans les discussions avec les banques ou les fournisseurs. En tant que partenaire de confiance, FMH Services vous assiste aussi dans ces discussions ou dans le choix du partenaire financier.

\section{Plan d'affaires}

Il vous faut élaborer un plan d'affaires car celuici sera impératif pour le financement étranger, outre le fait qu'il vous donnera une ligne de conduite dans la gestion de votre cabinet. Le plan d'affaires présente également l'avantage de vous contraindre à mener une réflexion systématique et précise concernant:

- vos idées et visions d'avenir;

- vos objectifs d'affaires qui serviront de paramètres pour évaluer les résultats futurs de votre cabinet;

- les chances et les risques de votre entreprise, ainsi que les lacunes éventuelles encore à combler.

Le plan d'affaires vous servira de base, ainsi qu'à vos partenaires d'affaires, pour évaluer votre projet de même que pour calculer vos chances de succès et le risque couru. Une fois ce plan établi, vous pourrez envisager avec confiance l'ouverture de votre propre cabinet!

\section{Vous n'êtes pas seul/e!}

Pour vos préparatifs, vous bénéficierez du soutien d'un expert-comptable expérimenté dans la gestion de cabinets médicaux (p.ex. FMH Fiduciaire Services). Avec vos partenaires de confiance, il vous conseillera durant la phase de préparation, dans la recherche d'un cabinet et dans les questions financières et mettra à votre disposition des contrats-modèles et des concepts d'assurance. FMH Services organise des séminaires gratuits sur l'ouverture d'un cabinet médical. Vous trouverez des informations à ce sujet et au sujet des partenaires de confiance de FMH Services sous www.fmhservices.ch. Nous vous recommandons aussi vivement de lire attentivement la brochure publiée par FMH Services sur la gestion d'un cabinet médical: «Reprise d'un cabinet médical: le chemin vers l'indépendance», qui vous sera remise gratuitement par FMH Consulting Services SA, Oberkirch.

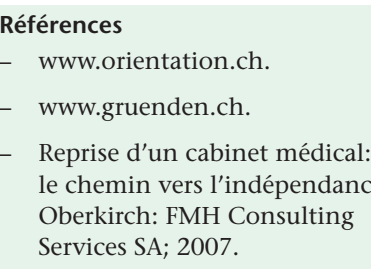

Reprise d'un cabinet médical: Oberkirch: FMH Consulting Services SA; 2007. 